\title{
Despegues sin madurez. Urbanización, industrialización y desarrollo en la Latinoamérica del siglo $\mathrm{XX}^{*}$
}

\author{
ARTURO ALMANDOZ* \\ ** Profesor Titular, Departamento de Planificación Urbana, Universidad Simón Bolívar.
}

\begin{abstract}
On the basis of some phases of Rostow's theory that explore the relationship between industrialization, urbanization and development, this article aims at illustrating how the imbalance between urbanization and the productive system helps to explain the issue of Latin America's takeoff throughout the twentieth century, lacking the ensuing drive to maturity. For that purpose, other political and social aspects are incorporated into a periodization that has to be initiated at the phase when Latin America started to evince a significant urbanization. Such a panoramic attempt is made from the historiographic and methodological premise that urbanization and development studies have often lost the historical perspective. It is therefore necessary to resume a panoramic and, to some extent, comparative review, both in historical and territorial terms, in which a long-term periodization will be a possible contribution of this research.
\end{abstract}

KEYWORDS: urbanization, development, Latin America, twentieth century

RESUMEN A partir de algunas fases de la teoría de Rostow que exploran la relación entre industrialización, urbanización y desarrollo, se busca ilustrar cómo el desbalance de la urbanización con respecto al aparato productivo explica en parte el despegue sin madurez de América Latina en el siglo XX. Para ello se incorporan otros aspectos políticos y sociales dentro de una periodización que se inicia cuando Latinoamérica comenzó a evidenciar una significativa urbanización. Esta apuesta panorámica se hace desde la premisa historiográfica y metodológica de que los estudios de urbanización y desarrollo han perdido la perspectiva histórica, por lo que hace falta retomar una posición panorámica y comparativa, tanto en términos históricos como territoriales.

PALABRAS CLAVE: urbanización, desarrollo, América Latina, siglo XX

\footnotetext{
Este artículo es otro avance del proyecto de investigación sobre "Modernización urbana en América Latina, 1850-2000", desarrollado por el autor desde 1999 en el departamento de Planificación Urbana y el Centro de Investigaciones Culturales de América Latina (CILCAL), Universidad Simón Bolívar (USB), Caracas. Una primera versión fue presentada en el evento "Construyendo la ciudad justa", Santiago de Chile: Pontificia Universidad Católica (PUC), marzo 2008.

Recibido el 25 de abril de 2008, aprobado el 15 de junio de 2008.

Correspondencia: Arturo Almandoz, Edificio MEU, Apartado 8900o, Caracas ro8o-A, Venezuela. E-mail: almandoz@usb.ve. Dirección electrónica: http://prof.usb.ve/almandoz.
} 


\section{Introducción}

En la entusiasta visión de la sociología funcionalista, la ecuación entre industrialización, urbanización y modernización — para utilizar el paradigma vigente hasta mediados de los sesenta- era una suerte de secuencia causal derivada de los exitosos casos de países industrializados y urbanizados a lo largo del siglo XIX y la primera mitad del XX (Reissman, 1970; Davis, 1982). Tal secuencia fue adoptada por algunos de los primeros estudios comparativos del proceso histórico latinoamericano, al menos en lo concerniente a la fase de incipiente industrialización y alto crecimiento urbano que se experimentaba en varios países desde la masificación de entreguerras (Hauser, 1967; Harris, 1971). Esa secuencia modernizadora se apoyaba, en gran medida, en las teorías del desarrollo económico de mediados del siglo XX, en especial la de Walt Whitman Rostow, las cuales daban un gran peso a la industrialización, el crecimiento y la estabilidad política dentro de la ecuación.

Para mediados de los sesenta, algunos países latinoamericanos eran vistos por Rostow y otros economistas como ejemplos que técnicamente habían iniciado el take-offo despegue al desarrollo. Tomando como indicador de éste más de 25 por ciento del Producto Nacional Bruto (PNB) destinado a inversión, ya Venezuela se había enrumbado en esta afortunada pista desde el frenesí progresista de los cincuenta, aventajando a Brasil, Colombia, Chile y Filipinas, países que le seguían en orden decreciente; si ya México y Argentina habían arrancado en la década anterior, el profesor norteamericano ciertamente señalaba a Venezuela y Brasil como los aviones de los sesenta (Rostow, 1990, pp. 44, 127).

Pero el desarrollo no venía dado sólo por un irreversible momento de despegue, sino que requería todo un largo y profundo proceso de cambios económicos, sociales y políticos, articulados en las fases recogidas por Rostow en The Stages of Economic Growth (1960), entre otros títulos de gran éxito. Combinando elementos de historia económica y política con caracterizaciones sociológicas, el profesor del Instituto Tecnológico de Massachusetts y otras universidades anglosajonas estudió y agrupó los casos de las “sociedades tradicionales" que, desde la Inglaterra de finales del siglo XVIII, habían tecnificado sus aparatos agrarios e industriales, entre otras condiciones, incrementando posteriormente el gasto en inversión requerido por el take-off, para posteriormente transitar un drive to maturity (empuje a la madurez), principalmente económica pero también social y política, que debía mantenerse por al menos dos generaciones de sostenido bienestar, antes de encontrar su vía hacia el desarrollo permanente. A partir de la gran diversidad de despegues a lo largo de los siglos XIX y XX — Francia y Estados Unidos en los años 1860, seguidos de Alemania en los setenta, Japón en el 1900, Rusia y Canadá en la primera posguerra, entre los más espectaculares casos_-, el mismo Rostow resumió las fases en el siguiente fresco que se cita:

Aquí, entonces, de una manera impresionista más que analítica, están los estadios de desarrollo que pueden ser distinguidos una vez que una sociedad tradicional comienza su modernización: el período transicional cuando las precondiciones para el despegue son creadas, generalmente en respuesta a la intrusión de un poder extranjero, convergiendo con ciertas fuerzas internas que contribuyen a la modernización; el despegue mismo; el empuje hacia la madurez, que generalmente abarca las vidas de dos generaciones más; y entonces, finalmente, si la elevación 
del ingreso ha igualado la difusión de virtuosismo tecnológico (...) la desviación de la economía completamente madura hacia la provisión de bienes de consumo duraderos y servicios (así como el Estado de Bienestar) para su población crecientemente urbana, y después suburbana (Rostow, 1990, p. 12). ${ }^{1}$

De manera que, entre otras condiciones de estabilidad política y modernización social, por cerca de cincuenta años después de despegar, los países ya referidos habían tenido que transitar un camino o empuje a la madurez o drive to maturity, caracterizado por dominio tecnológico en aquellos rubros con cuya industrialización habían decidido esas naciones ampliar la base económica de cara a asegurar el desarrollo; especialmente en los casos de pequeños territorios con limitados recursos naturales, como Suecia y Suiza, el país obviamente no tenía que autoabastecerse industrialmente, sino mostrar que disponía de "las habilidades técnicas y empresariales para producir no de todo, sino cualquier cosa que elige producir”. Hasta que esa madurez no se completara, lo cual se evidenciaba en la consolidación de una urbanizada sociedad de consumo masivo y un Estado de bienestar, los países que habían despegado seguían siendo considerados en desarrollo o developing countries, categoría que tenía mucha resonancia entonces para diferentes contextos, desde Taiwán y Corea del Sur hasta India y Turquía (Rostow, 1990, p. 10). ${ }^{2}$

Dentro del lote de países en desarrollo, en Latinoamérica destacaban los ya mencionados ejemplos de Brasil, Venezuela, Chile y Colombia; si bien ya para los años 1960 los casos de Argentina y México habían despegado para Rostow, la madurez no había sido alcanzada, y de hecho no lo sería por el resto del siglo. Resulta muy complejo explicar por qué no se obtuvo tal madurez en los países latinoamericanos, transcurridas varias décadas del fallido despegue. Tal como bien señalara el mismo autor, después del take-off había muchas decisiones que tomar y balances que mantener por parte de los países con respecto a las prioridades de desarrollo: la difusión de la tecnología moderna y el aumento de la tasa de crecimiento frente al incremento del consumo per cápita, así como la ampliación del gasto en bienestar social, sin caer en el excesivo crecimiento de la burocracia estatal, eran disyuntivas características del recorrido hacia la madurez, particularizadas y complejizadas por las coyunturas políticas y sociales que debía cada país enfrentar (Rostow, 1990, pp. 14-16).

A partir de esas fases de la teoría de Rostow que exploran la relación entre industrialización, crecimiento económico y desarrollo, la presente revisión trata de ilustrar cómo la urbanización y su desbalance con el aparato productivo, explica en parte la cuestión del despegue sin madurez en América Latina a lo largo del siglo XX. Ese intento ilustrativo debe estar atento asimismo

\footnotetext{
"Here then, in an impressionistic rather than an analytic way, are the stages-of-growth which can be distinguished once a traditional society begins its modernization: the transitional period when the preconditions for take-off are created generally in response to the intrusion of a foreign power, converging with certain domestic forces making for modernization; the take-off itself; the sweep into maturity generally taking up the lives of about two further generations; and then, finally, if the rise of income has matched the spread of technological virtuosity (...) the diversion of the fully mature economy to the provision of durable consumers' goods and services (as well as the welfare state) for its increasingly urban - and then suburban - population." (traducción del propio autor).

2 “...the technological and entrepreneurial skills to produce not everything, but anything that it choses to produce" (traducción del autor). Para los ejemplos de países en desarrollo, me apoyo también en Davis (1982).
} 
a detectar factores de la inaplicabilidad de la teoría rostowiana al contexto latinoamericano. Para todo ello se incorporan aspectos políticos y sociales dentro una periodización que es necesario iniciar con la fase en que Latinoamérica comenzó a evidenciar una significativa urbanización. Esta apuesta panorámica se hace desde la premisa de que los estudios de la urbanización y el desarrollo han perdido con frecuencia la perspectiva histórica (Clichevski, 1990; Drakakis-Smith, 1990, pp. 11-19; Potter y Lloyd-Evans, 1998, p. 28), por lo que hace falta retomar, desde la historiografía urbana, una revisión panorámica y hasta cierto punto comparativa, tanto en términos históricos como territoriales, que permita al mismo tiempo periodizar una visión de larga duración como una posible contribución de esta investigación (Almandoz, 2006). ${ }^{3}$

\section{Urbanización y masificación tempranas}

Ya para los años 1920, algunas regiones de América Latina albergaban dos habitantes en las ciudades por cada campesino que había permanecido en las pampas, llanos o sertones de su vasta geografía. Es un indicador muy grueso que oculta quizás contrastantes diferencias entre países: Argentina y el Cono Sur tenían más de 50 por ciento de su población urbanizada desde 1914, mientras que las repúblicas andinas y centroamericanas serían predominantemente rurales hasta los cincuenta (Beyhaut, 1985, pp. 210-211). A pesar de su relativa simplificación, los indicadores demográficos registraban una realidad inequívoca: disparado desde el mismo comienzo del siglo XX en algunos países, el proceso de urbanización sería indetenible en la mayor parte de Latinoamérica durante el segundo tercio del siglo. Y aunque sólo en términos demográficos, en pocas décadas se completaría un ciclo que había tomado más de una centuria en Gran Bretańa y otros países industrializados y urbanizados a lo largo del siglo XIX (Potter y Lloyd-Evans, 1998, pp. 9-11).

Como en otras regiones del más tarde llamado Tercer Mundo, la acelerada urbanización latinoamericana de comienzos del siglo XX acentuó las concentraciones metropolitanas dentro de un mapa en el que éstas contrastaban con la dispersión y el atraso rurales. Atiborradas de inmigrantes campesinos y foráneos, antiguas capitales coloniales y urbes emergentes pronto alcanzaron magnitudes que rivalizaban con metrópolis europeas y norteamericanas. Buenos Aires saltó de 663.000 habitantes en 1895 a 2.178 .000 en 1932; Santiago de 333.000 en 1907 a 696.000 en 1930; y Ciudad de México de 328.000 en 1908 a 1.049 .000 en 1933. Caso análogo al explosivo crecimiento de ciudades industriales como Manchester y Chicago, São Paulo pasó de 240.000 habitantes en 1900 a 579.000 en 1920 y 1.075 .000 en 1930; mientras que Río disminuyó su primacía urbana, con una población que sólo se incrementó de 650.000 habitantes en 1895 a 811.433 en 1906 (Geisse, 1987; Hardoy, 1988).

La expansión de las capitales fue en parte impulsada por un incipiente proceso de industrialización en Argentina, Uruguay, Chile y Cuba, los cuales figuraban entre los países más urbanizados del mundo para el primer cuarto de siglo. La población de La Habana se duplicó de 250.000 habitantes en 1900 a medio millón en 1925. Impulsadas por la migración del campo a la

\footnotetext{
3 Valga en este sentido señalar que, dado el propósito panorámico del artículo, en la bibliografía a referir se dará prioridad, por razones además de extensión del artículo, a las entradas generales o comparativas sobre América Latina, por sobre los casos de estudio nacionales o urbanos.
} 
ciudad, capitales de países andinos crecieron también de manera considerable: Bogotá pasó de 100.000 habitantes en 1900 a 330.000 en 1930, y Lima de 104.000 en 1891 a 273.000 en 1930. Aunque Caracas sólo creció de 72.429 habitantes en 1891 a 92.212 en 1920, los primeros efectos de la bonanza petrolera la harían pasar de 135.253 en 1926 a 203.342 en 1936 (Hardoy, 1997; Almandoz, 2002).

Las economías latinoamericanas seguían siendo principalmente agrarias o mineras, lo cual se evidenciaba todavía para el inicio de la depresión de 1929, con bajos porcentajes de participación industrial dentro del PNB de 22,8 en Argentina, 14,2 en México, 11,7 en Brasil y 7,9 en Chile, por citar los casos más elevados (Pozo, 2002, pp. 72-118). En la arena política, sin alcanzar el dramatismo de la Revolución Mexicana de 1910 — la cual fuera en parte desatada por el descuido hacia el campo feudal por parte de los gobiernos modernizadores de Porfirio Díaz (1877-80, 1884-1911) — los Estados latinoamericanos no pudieron prolongar el liberalismo decimonónico de corte positivista hasta el siglo XX, como el porfiriato, y más tarde Juan Vicente Gómez en Venezuela (1909-35), trataron de hacer. Desafiados por las demandas de sufragio universal, constitución de sindicatos y otros derechos políticos, los gobiernos de José Batlle y Ordóñez (1903-07, 1911-15) en Uruguay, Roque Sáenz Peña (1910-13) e Hipólito Irigoyen (1916-22) en Argentina, seguidos del primer Arturo Alessandri (1920-24, 1925) en Chile, ejemplificaron tempranos intentos del Estado posliberal por adaptarse a las demandas de la rápida urbanización (Baer y Pineo, 1998).

La relativa bonanza económica y la superación de los conflictos entre federalismo y centralismo, catalizados ambos por las demandas de la inmigración internacional desde finales del siglo XIX, permitieron a los países del Cono Sur girar el liberalismo político y económico hacia cuestiones sociales; en este sentido, las nuevas burguesías nacionales, aunque incipientes en términos de su base más comercial que industrial, lideraron reformas constitucionales que responderían a parte de los desafíos planteados por poblaciones más urbanas y heterogéneas. Así por ejemplo, el sufragio universal en la población masculina fue logrado en Argentina en 1912 y en Uruguay en 1919, más temprano que en algunos países europeos (Halperin Donghi, 2005, pp. 288326). Este tipo de reformas en países andinos y caribeños puede decirse demorado por factores varios, desde la persistencia de intestinos conflictos regionales, el inestable atractivo de tales países en circuitos internacionales de inversión, así como al carácter económicamente liberal pero políticamente conservador, tendente a autocrático, de prolongados regímenes como los de Augusto Leguía en Perú (1919-30), Gerardo Machado en Cuba (1925-33), así como el ya mencionado De Gómez en Venezuela (Caballero, 1994).

Muchas de las reivindicaciones de esa masa heterogénea — resultante para Romero de inmigrantes foráneos y provincianos que se mezclaban con sectores urbanos tradicionales pero venidos a menos (Romero, 1984, p. 337) — eran catalizadas por problemas habitacionales y sanitarios

\footnotetext{
$4 \quad$ El sentido de masa como cuerpo social heterogéneo y en trance de urbanización está tomado de José Luis Romero (1984, p. 337): "Fue la fusión entre los grupos inmigrantes y los sectores populares y de pequeńa clase media de la sociedad tradicional lo que constituyó la masa de las ciudades latinoamericanas a partir de los años de la primera guerra mundial. El nombre con que se la designó, más frecuente que el de multitud, adquirió cierto sentido restringido y preciso. La masa fue ese conjunto heterogéneo, marginalmente situado al lado de una so-
} 
en capitales socialmente explosivas, las cuales no podían seguir manteniendo, por razones tanto políticas como demográficas, sus deficiencias poscoloniales de servicios e infraestructura (Pineo y Baer, 1998). Respuestas oficiales y privadas a esas demandas configuraron la agenda urbana de las tres primeras décadas del siglo XX, especialmente en términos de reformas higiénicas y habitacionales de los centros históricos, completada por los suburbios residenciales para una burguesía que se tornaba cada vez más cosmopolita (Almandoz, 2002, pp. 28-31). Así, aunque la industrialización y el crecimiento económico fueran todavía insuficientes para el despegue, esa temprana agenda urbana de la masificación sentó las bases sociales de un incipiente Estado de Bienestar que era necesario para la madurez hacia el desarrollo, cuyas demandas más obvias no eran ya planteadas por la burguesía, sino más bien por esa masa en expansión.

\section{Desfase entre industrialización y urbanización}

Ya para 1950, más de la mitad de la población de Uruguay (78\%), Argentina (65,3\%), Chile $(58,4 \%)$ y Venezuela $(53,2 \%)$ vivía en centros urbanos. Mientras el promedio de urbanización en América Latina era todavía de 41,6\%, países como Brasil y México no eran demográficamente urbanizados sólo debido a la inmensa magnitud de sus poblaciones y territorios, albergando empero algunas de las mayores metrópolis del mundo (United Nations Centre for Human Settlements, 1996, p. 47). Ciudad de México y Río de Janeiro estaban apenas por debajo y por encima de los 3 millones, respectivamente, mientras São Paulo ya había escalado a 2 millones y medio. Este primer grupo de áreas metropolitanas latinoamericanas estaba todavía liderado por el Gran Buenos Aires, con 4,7 millones (Harris, 1971, p. 167).

Desde el final de la Segunda Guerra Mundial hasta mediados de los años 1960, las mayores economías latinoamericanas mostraron relativa prosperidad, marcada por un significativo crecimiento industrial por sustitución de importaciones, en medio de la ya mencionada urbanización. Impulsados por estos crecientes mercados urbanos a los que se dirigía la industrialización sustitutiva, México y Brasil alcanzaron un crecimiento anual del 6 por ciento, lo que los hizo calificar, para el mismo Rostow (1990, pp. 44, 127), como ejemplos del take-off o despegue desde los cincuenta y sesenta, respectivamente. Si bien los países del Cono Sur habían sido más dinámicos en el período de entreguerras, todavía mantenían un crecimiento de cuatro puntos (Clichevsky, 1990, pp. 22-23). Mientras tanto, liderados por los enormes ingresos de la Venezuela petrolera, el excedente rendido por la exportación de materias primas financió una segunda generación de sustitución de importaciones que también incluía a Colombia y Perú, en todos los cuales la participación industrial en el PNB era superior al 15 por ciento en 1955 , al tiempo que la tasa de crecimiento industrial duplicaba la del sector primario (Williamson, 1992, pp. 334-335; Pozo, 2002, p. 118).

Ese clima modernizador estaba penetrado de un nacionalismo económico compartido de manera heterodoxa por regímenes estatistas y liberales, democráticos y dictatoriales. Allí

ciedad normalizada, frente a la cual se presentaba como un conjunto anómico. Era un conjunto urbano, aunque urbanizado en distinta medida, puesto que se integraba con gente urbana de antigua data y gente de extracción rural que comenzaba a urbanizarse. Pero muy pronto su fisonomía fue decididamente urbana y lo fue su comportamiento...”. 
podrían incluirse desde los gobiernos populistas de Lázaro Cárdenas en México (1934-40), Juan Domingo Perón en Argentina (1946-55), y Getulio Vargas en Brasil (1934-45, 195054), hasta las dictaduras progresistas pero brutales de Fulgencio Batista en Cuba (1940-44, 1952-59) y Marcos Pérez Jiménez en Venezuela (1952-58). La agenda común de desarrollismo había sido respaldada, desde 1948, por la creación de agencias internacionales como la Organización de Estados Americanos (OEA) y la Comisión Económica para América Latina (CEPAL), patrocinadas por las Naciones Unidas y los crecientes intereses estadounidenses en la explotación primaria e industrial de la región. Con sede en Santiago de Chile y el liderazgo de Raúl Prebisch — antiguo Director del Banco Central de la República Argentina_, CEPAL fue piedra angular del desarrollismo latinoamericano de posguerra, basado en la sustitución de importaciones y otras políticas económicas que consolidaron el Estado corporativo en países considerados en desarrollo hasta mediados de los sesenta, cuando se agotara la asíllamada "fase fácil” de la sustitución de importaciones (Franco, 2007; Williamson, 1992, pp. 338-39).

Vistas entonces como prometedores ejemplos de developing countries, las sociedades latinoamericanas en trance de industrialización eran también consideradas como exponentes de la teoría clásica de modernización, según fuera concebida por el desarrollismo económico y la sociología funcionalista. Tal como ya fue señalado, desde comienzos de la década de los sesenta, la conexión entre industrialización, urbanización y modernización fue formulada, siguiendo una derivación casi causal, por Philip Hauser, Leonard Reissman y Kingsley Davis, desde la perspectiva de la transición demográfica y el consecuente cambio social, apoyándose para ello en los ejemplos de países del Atlántico Norte que se industrializaran en el siglo XIX (Reissman, 1970; Davis, 1982). De tal literatura pudo colegirse, sin embargo, que las naciones latinoamericanas en supuesto desarrollo parecían estar en el camino hacia la industrialización y urbanización, pero de hecho padecían profundas distorsiones en comparación con exitosas experiencias de modernización en Europa, Norteamérica y otras partes del mundo, tal como lo planteara Philip Hauser (1967).

Por un lado, la frágil industrialización no había precedido sino más bien seguido a la urbanización latinoamericana, de manera que la sustitución de importaciones no podía ser vista como equivalente de la "revolución industrial", con sus consiguientes efectos dinamizadores sobre el sistema económico y la transición demográfica (Drakakis-Smith, 1990, pp. 53-57; Williamson, 1992, p. 333). Tal como ocurriera en otras partes de lo que comenzaba a ser denominado Tercer Mundo, en lugar de haber "halado" ( $p$ ulled) hacia las ciudades contingentes poblacionales que pudieran ser de hecho absorbidos por la industria y otros sectores productivos, la mayor parte de la migración del campo a la ciudad latinoamericana había sido "empujada" (pushed) por un sector primario preterido por reformas agrarias demoradas o inexistentes, así como por políticas de énfasis urbano llevadas adelante por los Estados corporativos (Potter y Lloyd-Evans, 1998, pp. 12-13).

Por otro lado, los niveles de urbanización casi duplicaban la participación industrial en las economías argentina, chilena, venezolana, colombiana y brasileña, según los censos de los años 1950 (Harris, 1971, p. 85). Tales niveles no podían ser absorbidos por el sistema productivo, de manera que a la postre redundarían en "inflación urbana" o "superurbanización", tal como ocurriría en otras regiones del Tercer Mundo (Potter y Lloyd-Evans, 1998, pp. 14-15). En las 
décadas venideras, buena parte de este excedente de población improductiva viviendo en las ciudades terminaría alojada en barriadas y dependiendo de la economía informal. Pero era ya evidente para finales de los sesenta que el desbalance entre industrialización y urbanización no permitiría ni el desarrollo al estilo CEPAL, ni la modernización según la visión de la sociología funcionalista (Franco, 2007), así como tampoco la madurez en el sentido planteado por Rostow. Tal sobreurbanización ponía en duda la aplicabilidad de la teoría para los casos de despegue tardío en la segunda posguerra, al menos en una región de acelerada movilidad demográfica como Latinoamérica.

\section{El fracaso de la modernización y la respuesta marxista}

La revolución cubana de 1959, que sacó a Batista del poder e instaló el régimen marxista de Fidel Castro, prefiguró el clima político y económico de América Latina por el resto de la Guerra Fría. Para prevenir otras revoluciones de izquierda, la administración Kennedy decidió, con la asesoría de CEPAL, promover la así llamada Alianza para el Progreso, programa dirigido a consolidar la sustitución de importaciones, acelerar la reforma agraria y reducir las desigualdades sociales mediante la ayuda norteamericana a nuevas democracias de la región. Entre los beneficiarios estuvieron los gobiernos de Rómulo Betancourt en Venezuela (195964), Arturo Frondizi en Argentina (1958-62), Fernando Belaúnde Terry en Perú (1963-68), Eduardo Frei en Chile (1964-70) y, especialmente, los de Alberto Lleras Camargo (1958-62) y Carlos Lleras Restrepo (1966-70) en Colombia (Halperin Donghi, 2005, pp. 534-69).

A pesar de la Alianza para el Progreso y de la prolongada presencia de la sustitución de importaciones en las economías más grandes, para finales de los sesenta la industrialización no se había ni diversificado ni consolidado en Latinoamérica, especialmente en términos de bienes duraderos y de capital. La debilidad de la integración económica regional, la estrechez de algunos mercados nacionales y la desventaja de la mayoría de las manufacturas latinoamericanas para competir en circuitos internacionales —inundados ya en los setenta con productos made in Hong Kong, Taiwán y el resto del Lejano Oriente- son factores esgrimidos para explicar las restricciones estructurales de la sustitución de importaciones, algunos de los cuales pueden ser vistos como causas y efectos a la vez. Pero antes de su eventual fracaso, la "profundización" de este programa sustitutivo desde las manufacturas ligeras hasta las intermedias, y de allí hasta la maquinaria pesada, proceso que fue intentado hasta los setenta, agravó las distorsiones económicas y sociales del subdesarrollo latinoamericano.

Porque la profundización implicaba un cambio cuantitativo en la producción, desde la fase "fácil" de la sustitución de importaciones, que incluía manufactura con mano de obra intensiva, poco capacitada y de baja tecnología, hacia la fase "dura" de industrias capital-intensivas, de alta especialización y tecnología. El resultado fue que la creciente marea de fugitivos de la crisis en el campo no pudo ser absorbida por la industria en las ciudades, de manera que durante los años 1960 el desempleo urbano comenzó a aumentar (Williamson, 1992, pp. 339-40).

En efecto, para comienzos de los setenta era evidente que, más allá de la burguesía industrial y parte de la clase media, el "modernizante estilo de desarrollo" de las décadas anteriores no había alcanzado a otros estratos de la población, especialmente a la creciente masa de "pobreza 
urbana” que era engrosada por la migración rural-urbana (Clichevsky, 1990, p. 25). El fracaso del crecimiento económico, el desarrollismo y la modernización fue agravado, después de 1973, por los coletazos inflacionarios de las crisis internacionales, que en Latinoamérica no sólo fueron causadas por los elevados precios de los combustibles, sino también por el impagable incremento de la maquinaria importada del mundo industrializado. Empeorado por el socialismo y las guerrillas marxistas al estilo cubano, tanto rurales como urbanas, que recrearon en términos revolucionarios el atávico mito del buen salvaje (Rangel, 2005), el malestar económico y social llevó a algunas de las más estables democracias latinoamericanas a abrazar dictaduras que marcarían su evolución hasta finales de los ochenta, tal como fue tempranamente dramatizado por el Chile de Augusto Pinochet (1973-90).

El agotamiento de la sustitución de importaciones fracturó así el soporte que la industrialización había provisto en la posguerra al proceso de urbanización, cuyo nivel se incrementó de 57,4 a 65,4 por ciento en Latinoamérica en su conjunto (Clichevsky, 1990, p. 42). Muy por encima de África y Asia — que estaban todavía en 28,7 y 26,6 para 1980, respectivamenteAmérica Latina era la región más urbanizada de lo que, más que "en desarrollo", era ya conocido como el Tercer Mundo (Drakakis-Smith, 1990; Potter y Lloyd-Evans, 1998, pp. 24-25). Este síndrome urbano subdesarrollado ha sido caracterizado por Potter y Lloyd-Evans en los siguientes términos: "como los estándares de salud y el bienestar social generalmente son mucho mejores en las ciudades que en las áreas rurales, las ciudades del Tercer Mundo ejemplifican por excelencia la combinación de fertilidad pre-industrial con la mortalidad posindustrial. Las ciudades contemporáneas en el mundo en desarrollo exhiben algunas de las más altas tasas de crecimiento natural encontrables en ciudades" (Potter y Lloyd-Evans, 1998, p. 12).

En efecto, a lo largo de los años 1970, las tasas latinoamericanas de crecimiento urbano eran seis veces mayores que las rurales (Clichevsky, 1990, p. 48), lo que confirmaba las ingentes corrientes que arribaban a las ciudades desde el campo. Para colmo de males, la mayor parte de esta población estaba altamente concentrada en los territorios nacionales: no sólo tenía Latinoamérica tres de las cinco megalópolis por encima de 8 millones en 1970 (Clark, 2000, p. 46), sino que para 1980 más de la mitad de las poblaciones nacionales de Argentina, Brasil, México, Venezuela, Chile y Colombia vivía en áreas metropolitanas superiores a $100 \mathrm{mil}$ habitantes (Clichevsky, 1990, p. 54).

Desde los setenta Latinoamérica exhibía así los efectos más dramáticos de la sobreurbanización, tales como la hipertrofia del sector terciario y de la economía informal que camuflaba el excedente de fuerza de trabajo en las ciudades, completada con la proliferación de asentamientos no controlados y pobreza. El fracaso del desarrollismo y la modernización, junto al consiguiente síndrome de la urbanización del Tercer Mundo, condenaron el enfoque funcionalista de CEPAL que había prevalecido hasta los sesenta; quedaba así atrás la diversificación y tecnologización de los sectores productivos implicados en la madurez rostowiana, desbordados por contingentes urbanos subcalificados que abultaban los aparatos administrativos. Aunque fuera prefigurado desde la década anterior, se hacía evidente desde los setenta que las fases del desarrollo de Rostow y la modernización funcionalista eran partes de un paradigma económico y social que, más que inalcanzable, resultaba agotado e inválido dentro del clima político, técnico y académico de América Latina. 
En parte concebida como alternativa a la doctrina liberal de las ventajas comerciales comparativas, la cual había explicado tradicionalmente el atraso latinoamericano en la economía mundial desde finales de la Colonia (Morse, 1975), la teoría de la Dependencia reinterpretó la antinomia centro/periferia como una rémora estructural que sólo podría ser superada sobre la base keynesiana de intervención pública estatal (Williamson, 1992, pp. 334-35). En este sentido, el enfoque de la Dependencia no estaba originalmente opuesto a las iniciativas cepalinas, incluyendo la sustitución de importaciones; pero en la medida en que esta última probó estar agotada, el dependentismo devino una alternativa predominantemente marxista frente al desarrollismo capitalista. Con contribuciones ulteriores de los brasileńos Celso Furtado, Fernando H. Cardoso y el chileno Enzo Faletto, entre otros, la teoría de la Dependencia se convirtió en una suerte de escuela de ciencias sociales, la cual proveyó una matriz histórica que buscaba explicar el atraso colonial y republicano de América Latina, incluyendo dimensiones económicas, políticas y sociales del subdesarrollo secular (Cardoso y Faletto, 1969; Palma, 1978).

Marcando el clima intelectual y académico hasta comienzos de los ochenta, la escuela de la Dependencia reforzó también las tendencias nacionalistas de gobiernos opuestos a la presencia de capitales foráneos en la explotación de materias primas y procesos industriales (Pozo, 2002, pp. 177-179). Al mismo tiempo, los efectos económicos y urbanos del agotamiento del estadio "fácil" de la sustitución de importaciones fueron resumidos por teóricos de la Dependencia en los siguientes términos: esta primera fase del proceso de sustitución, llamada "fácil", porque arrojó rápidos y visibles ritmos de crecimiento industrial, condujo a la formación de una base manufacturera liviana y, en consecuencia, al surgimiento de una burguesía industrial y de un proletariado de cierta consideración que se concentró principalmente en los centros urbanos. El sistema, no obstante el rápido crecimiento, se vio incapacitado de absorber la población excedente que procedía de las áreas rurales y era generada por la penetración en la agricultura de relaciones capitalistas de producción con la consecuente destrucción de los sectores productivos no capitalistas. El resultado fue la concentración en las ciudades de una masa que ha sido descrita empíricamente como "marginal" (Malavé Mata, Silva Michelena y Sonntag, 1979, p. 93).

\section{La década perdida y el neoliberalismo}

El agotamiento de la sustitución de importaciones, las crisis petroleras y recesiones de los setenta, la disminución de las exportaciones latinoamericanas dentro del total mundial — que habían pasado del 13,5 por ciento en 1946 a 5,1 en 1970 (Pozo, 2002, p. 176) — agravaron así el endeudamiento externo, que había amenazado a las repúblicas latinoamericanas desde su independencia. A pesar de la renuencia del desarrollismo nacionalista para aceptar capital foráneo, los empréstitos de la banca privada internacional se incrementaron como en ninguna otra región del mundo, a tal punto que éstos representaban más del 50 por ciento del PNB de América Latina para 1980, así como de las mayores economías, con la excepción de Brasil. Mientras tanto, las desaceleradas tasas de crecimiento del PNB nacional hicieron que el promedio regional decayera de 4,5 en los setenta a 1,3 en la década siguiente, cuando la hiperinflación superó el 2.000 por ciento anual en Argentina, Brasil y Perú, entre los casos más dramáticos (Pozo, 2002, pp. 179-180). Sólo Jamaica, Colombia y Chile escaparon de 
un declive general en el ingreso per cápita durante el período (United Nations Centre for Human Settlements, 1996, p. 45). Es por todo ello que los años 1980 pasaron a ser conocidos como la "década perdida" de Latinoamérica, especialmente por contraste con el espectacular desarrollo alcanzado por los así llamados “tigres” asiáticos —Corea del Sur, Singapur, Malasia y Tailandia-, así como por Espańa y otros nuevos miembros de la entonces Comunidad Europea (CE), cuya evolución, vista en perspectiva desde los cincuenta, confirmaba en general las fases de Rostow.

Poniendo en evidencia el agotamiento del "modelo latinoamericano de desarrollo" — que se había caracterizado desde los ańos 1930 por una agricultura improductiva y subordinada a la industria protegida, así como por una fuerte presencia del Estado corporativo ${ }^{5}$ — la década perdida implicó una nueva y creciente dependencia de agencias como el Fondo Monetario Internacional (FMI) y el Banco Mundial (BM), los cuales dictaron desde entonces las recomendaciones o recetas económicas y sociales a ser seguidas por los endeudados países. Inspirados por la Nueva Derecha del eje anglo-americano, ${ }^{6}$ los "planes de ajuste" firmados desde 1982 fueron de hecho paquetes de políticas neoliberales, incluyendo la reducción de las burocracias mostrencas y la privatización de muchos servicios y compañías de los Estados corporativos latinoamericanos. Con el asesoramiento de Milton Friedman y la escuela de los Chicago boys, que incluyó una renovación de la agroindustria para la exportación, el Chile de Pinochet fue un temprano éxito demostrativo de que las reformas podían ser emprendidas por regímenes autoritarios, como habían sido en parte los casos de Corea del Sur y la España del último Franco.

El paquete neoliberal fue aplicable, aunque produciendo inestabilidad a largo plazo, en las administraciones de Carlos Salinas de Gortari (1988-94) en México y Carlos Menem (198995, 1995-99) en Argentina, especialmente durante su primer mandato. Pero las reformas requeridas por el FMI y el BM probaron ser desastrosas cuando fueron introducidas demasiado tarde y drásticamente después de una relativa bonanza, tal como ocurrió durante el segundo gobierno de Carlos Andrés Pérez (1989-93) en Venezuela, marcado por saqueos, malestar social y golpes militares. El violento clima político y social venezolano fue exportado a otros países latinoamericanos donde, empeorado por el así llamado "efecto tequila" en México en 1994, los ajustes neoliberales no pudieron disminuir las desigualdades sociales, sino más bien incrementaron la pobreza y criminalidad para finales de la década (Rotker, 2000).

\footnotetext{
5 Tal como lo resume Del Pozo (2002, p. 182): “Todos estos elementos llevaban, a fines de los 1980, a preguntarse si el modelo latinoamericano de desarrollo, creado entre 1930 y 1950, basado en la prioridad de una industrialización que se desarrollaba tras murallas proteccionistas, a la cual la agricultura se había subordinado, y que contaba con una presencia importante del Estado, había llegado a un callejón sin salida. Esa había sido sin duda la percepción de los militares y de los empresarios que diseñaron la política económica de las dictaduras, especialmente en el Cono Sur desde los años 1970, que condujo, en países como Argentina y Chile, a una reorientación de la economía, donde se daba prioridad a las actividades de exportación de productos primarios, en detrimento de la industria, que ponía énfasis en la privatización de las empresas del Estado y que se basaba en una represión implacable contra los sindicatos y cualquier otra organización de trabajadores".

6 Nos referimos, por supuesto, al eje definido por las reformas neoliberales durante los gobiernos de Ronald Reagan en Estados Unidos (1981-89) y Margaret Thatcher (1979-90) en Gran Bretaña.
} 
La privatización y el desmantelamiento de los aparatos públicos, que habían sobreterciarizado varias economías latinoamericanas, dispararon niveles de desempleo superiores al 20 por ciento en la primera mitad de los ochenta, con alarmantes crecimientos de economía informal que resultaban peores a los de la crisis de 1929. Es cierto que el peso del pago de la deuda se aminoraría para la década de los noventa, cuando en muchas de las economías pasó a ser menos del 25 por ciento del valor de las exportaciones, mientras el PIB superaba los tres puntos. Sin embargo, se produjo un agravamiento de las desigualdades sociales en casi todos los países grandes de la región, donde el quintil más rico de la población — acaparando sobre el 40 por ciento del ingreso- era más de 15 veces superior al quintil más pobre para finales de los noventa (Pozo, 2002, pp. 181, 237-238, 242).

Con 71,4 por ciento de su población viviendo en asentamientos urbanos para 1990, Latinoamérica está desde entonces completando su ciclo de urbanización, flirteando con un neoliberalismo que trató de paliar la recesión de la década perdida, seguido de una resurrección izquierdista que se ha opuesto a las desigualdades generadas por esas políticas que han pasado a ser percibidas como neoimperialistas (Irazábal, 2008). El crecimiento más lento de la población fue en parte consecuencia de una fertilidad y migración rural-urbana más bajas, lo que resultó en menores tasas de crecimiento para muchas de las mayores ciudades latinoamericanas. ${ }^{7} \mathrm{La}$ culminación del ciclo de urbanización demográfica en países como Argentina, Uruguay, Chile y Venezuela no debe ser confundida empero con la corrección de los desbalances territoriales o de las distorsiones económicas, sin desmerecer el milagro chileno. Un indicador de la excesiva concentración urbana latinoamericana puede verse en que, albergando 29 por ciento de la población en 1990, había más habitantes en ciudades millonarias que en áreas rurales (United Nations Centre for Human Settlements, 1996, pp. 42-43, 47-48).

La fuerte atracción de las ciudades millonarias por sobre las metrópolis tradicionales es debida al agotamiento de estas últimas en términos de una infraestructura desgastada y un deterioro de los niveles de vida, lo que ha llevado a su vez a un giro de la migración rural-urbana a la interurbana (Clichevsky, 1990, p. 47; De Mattos, 2006). Las ciudades medianas de algunos de los países latinoamericanos más urbanizados han sido favorecidas por ese giro, especialmente en lo concerniente a profesionales de clase media que buscan vivienda y otros servicios que resultan inasequibles en las metrópolis. Aunque la relativa desconcentración puede ser vista como un efecto positivo del fin del ciclo urbanizador, esa movilidad refleja en el fondo el empobrecimiento de la clase media, afectada por el declive del ingreso per cápita y del salario

\footnotetext{
Es conveniente diferenciar finalmente este panorama general según los niveles de urbanización de los países para mediados de los noventa (United Nations Centre for Human Settlements, 1996, p. 48): "Aunque la exactitud de comparaciones entre países en sus niveles de urbanización están siempre sujetas a las diferencias de criterios usados para definir centros urbanos, es posible distinguir tres grupos de naciones. El primero, los más urbanizados, con más de 80 por ciento de sus poblaciones viviendo en areas urbanas, incluye las tres naciones en el Cono Sur y Venezuela. Con entre 50 y 80 por ciento en areas urbanas, el segundo incluye la mayoría de los países que tuvieron un rápido desarrollo industrial en el período 1950-90 — República Dominicana, México, Brasil, Ecuador y Colombia - y también Cuba (que ya era una de las naciones más urbanizadas de la región en 1950), Bolivia, Perú, Nicaragua, Jamaica y Trinidad y Tobago. Con menos del 50 por ciento de la población en áreas urbanas, el tercero incluye sólo uno en Suramérica (Paraguay) y uno en el Caribe (Haití), junto a un grupo de países en América Central (Costa Rica, El Salvador, Guatemala y Honduras)...” (traducción del autor).
} 
real. Para finales de los noventa, las tasas de desempleo frisaban el 15 por ciento en Argentina, Colombia, Ecuador, Jamaica, República Dominicana y Venezuela, mientras la proporción de hogares pobres era de 36 para el conjunto regional, agravándose hasta 40 en la crisis de 2001 en Argentina, otrora paraíso de la clase media latinoamericana (Pozo, 2002, pp. 240-241).

La diversificación de la economía informal y el agravamiento de la pobreza han tenido efectos dramáticos en la escena urbana, especialmente en términos de la invasión del espacio público por vendedores ambulantes y el establecimiento de gated communities tanto en distritos residenciales como en barriadas no controladas. La segregación de la ciudad dual ha estado acompañada del deterioro de la infraestructura y el incremento de pobreza urbana, que para 1990 alcanzaba 40 por ciento en Colombia, 38 en Brasil, 28 en Venezuela y 23 en México (United Nations Centre for Human Settlements, 1996, p. 528). Criminalidad, inestabilidad social y falta de gobernabilidad han permanecido como problemas nacionales, cuyos escenarios más dramáticos han sido las áreas metropolitanas tensionadas por grupos en conflicto (Villasante, 1994; Rotker, 2000; Irazábal, 2008). Una agenda de malestar económico y social para un continente que ha completado su ciclo de urbanización después de más de un siglo, parte de cuyo accidentado proceso puede ser visto en relación con las fases de Rostow, tal como se intentará recapitular a continuación para concluir.

\section{Comentarios finales}

No haber alcanzado la madurez después de un despegue que fue anunciado por el mismo Rostow es atribuible a diversas e innumerables causas, según los particulares procesos de los países latinoamericanos; sin embargo, al tratar de establecer las razones más generales, se puede señalar primeramente la inestabilidad política que no permitió la consolidación del Estado de bienestar difusor de beneficios sociales. Ello fue tempranamente evidenciado por Argentina — primer país llamado al despegue_- después del golpe de Uriburu a Irigoyen en 1932, cuyo régimen había promovido reivindicaciones sociales que respondían a la masificación urbana. A pesar de su avance en las primeras décadas, para los años 1950 el país estaba endeudado por el excesivo gasto social del populismo peronista y se adentraba, en vez de la madurez, en la turbulencia de las dictaduras subsiguientes, por lo que Argentina comenzaba a ser vista como el primer desarrollo frustrado en la Latinoamérica del siglo XX.

El agotamiento de la sustitución de importaciones y otros programas económicos como la reforma agraria también pesaron, como causas y efectos a la vez, en lo que puede ser llamado la inmadurez del desarrollo latinoamericano - eufemismo resultante de las fases rostowianas que pasaría a ser parte del subdesarrollo mismo (Sunkel, 1973)—. En efecto, tal como fue señalado, ya para finales de los años 1960 era evidente que la industrialización no se había ni diversificado ni consolidado, especialmente en términos de bienes de consumo duraderos y de capital, con lo que quedaban estranguladas las posibilidades de tecnificación de la madurez rostowiana. A pesar de los relativos avances en los sesenta del Mercado Común Centroamericano (MCC), la Asociación Latinoamericana de Libre Comercio (ALALC, después ALADI), y del Pacto Andino desde 1969, el debilitamiento de la industrialización latinoamericana fue confirmado en la decreciente participación de su manufactura en el mercado mundial hasta los años 1970 . 
Aun cuando la periodización aquí desarrollada ha tratado de cubrir todo el siglo XX, puede decirse que el desbalance que se produjo entre industrialización y urbanización en Latinoamérica después del agotamiento de aquélla, con la consecuente inflación urbana y sobreterciarización, puso fin a las posibilidades de la madurez para el desarrollo en términos de las fases y la lógica de Rostow. Porque el proceso latinoamericano a partir de los setenta, tal como lo evidenció desde temprano la dictadura de Pinochet en Chile, revisaría esa industrialización patrocinada por el Estado corporativo, de cara a tecnificar otros sectores más competitivos en el mercado mundial, así como disminuir el gasto público en servicios. Ese fue un punto de quiebre del modelo latinoamericano que, desde la masificación de los años 1930, podía ser todavía medible en términos de las fases rostowianas, las cuales son sólo alcanzables sobre la base de la industrialización.

Pero con nuevos horizontes tecnológicos y geopolíticos, así como un nuevo paradigma de desarrollo sustentable (Gabaldón, 2008), a partir de los ochenta tuvieron que ser adoptados otros modelos económicos, como el neoliberalismo hasta finales de siglo XX, o incluso el nuevo corporativismo estatal de comienzos del XXI, cuyas respuestas al desafío del desarrollo están aún por verse. Sin embargo, creemos que las fases de la teoría de Rostow proveyeron, al menos hasta los setenta, una matriz para poner el proceso urbano y económico de Latinoamérica en perspectiva histórica, aunque su interés ahora pueda ser más bien historiográfico, como este artículo ha tratado de mostrar.

\section{Referencias bibliográficas}

Almandoz, A. (2002). Urbanization and urbanism in Latin America: from Haussmann to CIAM. En A. Almandoz (Ed.), Planning Latin America's capital cities, 1850-1950 (1a ed., pp. 13-44). Londres y Nueva York: Routledge.

Almandoz, A. (2006). Urban planning and historiography in Latin America. Progress in Planning, 65, 2, 81-123.

Baer, R. \& Pineo, J. A. (1908). Introduction. En R. Pineo \& J. A. Baer (Eds.), Cities of hope. People, protests and progress in urbanizing Latin America, 1870-1930 (1a ed., pp. 7-14). Boulder: Westview Press.

Beyhaut, G. H. (1985). Historia universal siglo XXI. América Latina. III. De la independencia a la segunda guerra mundial (1 ${ }^{a}$ ed., Vol 23). México: Siglo Veintiuno Editores.

Caballero, M. (1994). Gómez, el tirano liberal (2a ed.). Caracas: Monte Ávila Editores Latinoamericana.

Cardoso, F. H. \& Faletto, E. (1969). Dependencia y desarrollo en América Latina (1a ed.). México: Siglo XXI.

Clark, D. (2000). Urban world / Global city (1 $\left.1^{\mathrm{a}} \mathrm{ed}.\right)$. Londres: Routledge.

Clichevsky, N. (1990). Construcción y administración de la ciudad latinoamericana (1a ed.). Buenos Aires: Instituto Internacional de Medio Ambiente y Desarrollo (IIED-América Latina), Grupo Editor Latinoamericano (GEL).

Davis, K. (1982). La urbanización de la población mundial. En AA.VV., La ciudad (4a ed., pp. 11-36). Madrid: Scientific American, Alianza Editorial.

De Mattos, C. (2006). Modernización capitalista y transformación metropolitana en América Latina: cinco tendencias constitutivas. En A. I. G. Lemos, M. Arroyo \& M. L. Silveira 
(Eds.), America Latina: cidade, campo e turismo (1a ed., pp. 41-73). Buenos Aires: CLACSO-Universidad de San Pablo.

Del Pozo, J. (2002). Historia de América Latina y del Caribe. 1825-2001 (1 a ed.) Santiago: LOM Ediciones.

Drakakis-Smith, D. (1990). The third world city (1a. ed.). Londres: Routledge.

Franco, R. (2007). La FLACSO clásica (1957-1963): vicisitudes de las ciencias sociales latinoamericanas ( $\left.1^{\mathrm{a}} \mathrm{ed}.\right)$. Santiago de Chile: Catalonia.

Gabaldón, A. J. (2008). Desarrollo sustentable. La salida de América Latina (1ª ed.). Caracas: Grijalbo.

Geisse, G. (1987). Tres momentos históricos en la ciudad hispanoamericana del siglo XIX. En G. Alomar (Coord.), De Teotihuacán a Brasilia. Estudios de historia urbana iberoamericana y flipina ( $1^{\mathrm{a}}$ ed., pp. 397-433). Madrid: Instituto de Estudios de Administración Local (IEAL).

Halperin Dongui, T. (2005). Historia contemporánea de América Latina (6a ed.). Madrid y Buenos Aires: Alianza Editorial.

Hardoy, J. E. (1988). Teorías y prácticas urbanísticas en Europa entre 1850 y 1930. Su traslado a América Latina. En J. E. Hardoy \& R. M. Morse (Eds.), Repensando la ciudad de América Latina (1a ed., pp. 97-126). Buenos Aires: Grupo Editor Latinoamericano (GEL).

Hardoy, J. E. (1997). Las ciudades de América Latina a partir de 1900. En AA. VV., La ciudad hispanoamericana. El sueño de un orden (2a ed., pp. 267-274). Madrid: Centro de Estudios Históricos de Obras Públicas y Urbanismo (CEHOPU), Centro de Estudios y Experimentación de Obras Públicas (CEDEX), Ministerio de Fomento.

Harris, W. D. Jr. (1971). The growth of Latin American cities (1a. ed.) Athens, Ohio: Ohio University Press.

Hauser, P. M. (Ed.) (1967). La urbanización en América Latina (2a. ed.). Buenos Aires: Solar, Hachette.

Irazábal, C. (2008). Citizenship, democracy and public space in Latin America. En C. Irazábal (Ed.), Ordinary places, extraordinary events. Citizenship, democracy and public space in Latin America (1a ed., pp. 11-34). Londres y Nueva York: Routledge.

Malavé Mata, H.; Silva Michelena, H. \& Sonntag, H.R. (1979). El contenido conflictivo del actual proceso económico-social de América Latina (pp. 92-105). En AA. VV., Ensayos venezolanos (1a ed., pp. 92-122). Caracas: Ateneo de Caracas.

Morse, R. M. (1975). El desarrollo de los sistemas urbanos en las Américas durante el siglo XIX. En J. E. Hardoy \& R. P. Schaedel (Eds.), Las ciudades de América Latina y sus áreas de influencia a través de la historia (1a ed., pp. 263-290). Buenos Aires: Sociedad Interamericana de Planificación (SIAP).

Palma, G. (1978). Dependency: a formal theory of underdevelopment or a methodology for the analysis of concrete situations of underdevelopment. World Development, 7/8, $881-920$.

Pineo, R. \& Baer, J. A. (1998). Urbanization, the working class and reform. En R. Pineo \& J. A. Baer (Eds.), Cities of hope. People, protests and progress in urbanizing Latin America, 1870-1930 (1a. ed., pp. 258-274). Boulder: Westview Press.

Potter, R. B. \& Lloyd-Evans, S. (1998). The city in the developing world (1a ed.) Londres: Longman. 
Rangel, C. (2005). Del buen salvaje al buen revolucionario. Mitos y realidades de América Latina ( $4^{\mathrm{a}} \mathrm{ed}$.). Caracas: Criteria.

Reissman, L. (1970). The urban process. Cities in industrial societies (2a. ed.). Nueva York: The Free Press.

Romero, J. L. (1984). Latinoamérica: las ciudades y las ideas (3a ed.). México: Siglo Veintiuno.

Rostow, W. W. (1990). The stages of economic growth. A non-communist manifesto (2a. ed.). Nueva York: Cambridge University Press.

Rotker, S. (Ed.) (2000). Ciudadanías del miedo (1ª ed.). Caracas: Nueva Sociedad, Rutgers University.

Sunkel, O. (1973). El subdesarrollo latinoamericano y la teoría del desarrollo (6a ed.). México: Siglo Veintiuno Editores.

United Nations Centre for Human Settlements (HABITAT) (1996). An urbanizing world. Global report on human settlements (1a ed.). Oxford: Oxford University Press.

Villasante, T. R. (Ed.) (1994). Las ciudades hablan. Identidades y movimientos sociales en seis metrópolis latinoamericanas ( $1^{\text {a }}$ ed.). Caracas: Nueva Sociedad.

Williamson, E. (1992). The Penguin history of Latin America (1 ${ }^{\mathrm{a}}$. ed.). Londres: Penguin Books. 